\title{
Selective N,N-Dimethylation of Primary Aromatic Amines with Methyl Alkyl Carbonates in the Presence of Phosphonium Salts.
}

Maurizio Selva, * Alvise Perosa, Pietro Tundo, and Davide Brunelli

Dipartimento di Scienze Ambientali dell’Università Ca’ Foscari, and

Consorzio Interuniversitario “La Chimica per l’Ambiente” (INCA), UdR di Venezia

Calle Larga S. Marta, 2137 - 30123 - Venezia, Italy

\section{Supporting Information}

- General Experimental Methods $\quad$ S2

- Isolation and characterisation of methyl alkyl carbonates $\quad$ S2

- ${ }^{1}$ H NMR spectrum of 2-[2-(2-Methoxyethoxy)ethoxy]ethyl methyl carbonate, 1a $\quad$ S2

- ${ }^{13} \mathrm{C}$ NMR spectrum of 2-[2-(2-Methoxyethoxy)ethoxy]ethyl methyl carbonate, 1a $\mathrm{S} 3$

- $\quad{ }^{1} \mathrm{H}$ NMR spectrum of 2-(2-Methoxyethoxy)methylethyl carbonate, 1c S3

- ${ }^{13}$ C NMR spectrum of 2-(2-Methoxyethoxy)methylethyl carbonate, 1c S4

- Isolation and characterisation of N,N-dimethyl anilines $\left(\mathbf{D}_{\mathbf{x}}\right) \quad \mathrm{S} 5$

- ${ }^{1} \mathrm{H}$ NMR of N,N-dimethyl $p$-anisidine $\quad$ S5

- ${ }^{1} \mathrm{H}$ NMR of N,N-dimethyl $p$-toluidine $\quad$ S6

- ${ }^{1} \mathrm{H}$ NMR of N,N-dimethylaniline $\quad$ S7

- ${ }^{1} \mathrm{H}$ NMR of N,N-dimethyl p-chloroaniline $\quad$ S7

- ${ }^{1} \mathrm{H}$ NMR of methyl N,N-dimethylaminobenzoate $\quad$ S8

- ${ }^{1} \mathrm{H}$ NMR of N,N-dimethyl o-ethylaniline $\quad$ S8

- $\quad{ }^{1} \mathrm{H}$ NMR of $N, N$-dimethyl 2,3-dimethylaniline $\quad$ S9

- $\quad$ GC/MS of N-Ethyl,N-methyl $m$-toluidine, $7 \mathbf{b} \quad S 9$

- Overlap of IR spectra of $\mathbf{5 b}, \mathbf{3 b}$ and the mixture $\mathbf{5 b} / \mathbf{3 b} \quad \mathrm{S} 10$

- Overlap of IR spectra of 5a, 3a and the mixture 5a/3a $\quad$ S11

- References $\quad \mathrm{S} 12$ 
General Experimental Methods. GLC and GC/MS (70 eV) analyses were run using HP5 and HP5/MS capillary columns (30 m), respectively. ${ }^{1} \mathrm{H}$ and spectra were recorded at 300 and $400 \mathrm{MHz}$ spectrometers, ${ }^{13} \mathrm{C}$ NMR at 75 and $100 \mathrm{MHz}$. Chemical shifts are reported in $\delta$ values downfield from TMS. $\mathrm{CDCl}_{3}$ was used as the solvent. IR spectra were recorded at room temperature on $\mathrm{KBr}$ pellets.

Compounds 3a-d, 4a-b, 5a-g, 6a-b, 7a, DMC, and $\mathrm{K}_{2} \mathrm{CO}_{3}$ were ACS grade and were employed without further purification.

\section{Isolation and characterisation of methyl alkyl carbonates.}

Methyl alkyl carbonates 1a-c were purified by distillation under vacuum, and recovered as colorless liquids: 1a (b.p. $\left.92{ }^{\circ} \mathrm{C} / 100 \mathrm{~Pa}\right) 72 \%$ yield (98\% purity by GC); $\mathbf{1 b}$ (b.p. $65{ }^{\circ} \mathrm{C} / 100 \mathrm{~Pa}$ ) $62 \%$ yield (99.5\% purity by GC); 1c (b.p. $\left.39{ }^{\circ} \mathrm{C} / 100 \mathrm{~Pa}\right) 60 \%$ yield (98.5\% purity by GC). Full spectroscopic data of $\mathbf{1 b}$ were already reported: ${ }^{1}$ the structure of $\mathbf{1 b}$ was confirmed by comparison to an authentic sample. 1a and 1c were characterized by GC/MS, ${ }^{1} \mathrm{H}$ NMR, and ${ }^{13} \mathrm{C}$ NMR.

2-[2-(2-Methoxyethoxy)ethoxy]ethyl methyl carbonate, 1a. ${ }^{1} \mathrm{H}$ NMR $\left(400 \mathrm{MHz}, \mathrm{CDCl}_{3}\right) \delta$ 4.29-4.25 (m, 2H), 3.76 (s, 3H), 3.72-3.68 (m, 2H), 3.67-3.60 (m, 6H), 3.55-3.51 (m, 2H), 3.36 (s, 3H). ${ }^{13} \mathrm{C}$ NMR $\delta\left(100 \mathrm{MHz}, \mathrm{CDCl}_{3}\right), 54.9,69.0,67.0,68.9,70.51,70.55,70.6,71.9,155.7 . \mathrm{GC}-$ MS, 70 eV, m/z: $222\left(\mathrm{M}^{+},<1 \%\right), 103$ (100), 89 (12), 59 (76), 58 (27). Anal. Calcd. for $\mathrm{C}_{9} \mathrm{H}_{18} \mathrm{O}_{6}$ : C, 48.65; H, 8.11. Found: C, 48.72; H, 8.19. 


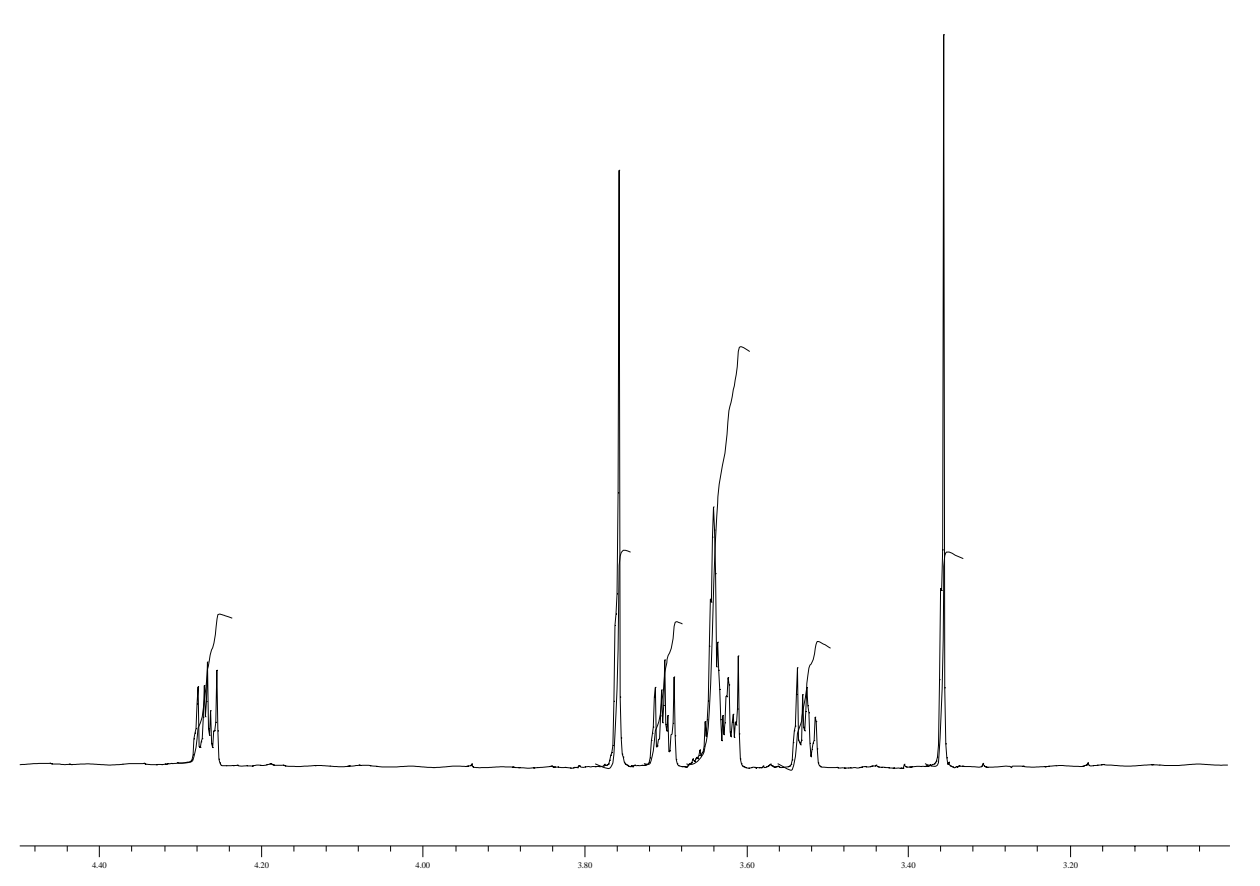

Figure 1. ${ }^{1} \mathrm{H}$ NMR of carbonate 1a

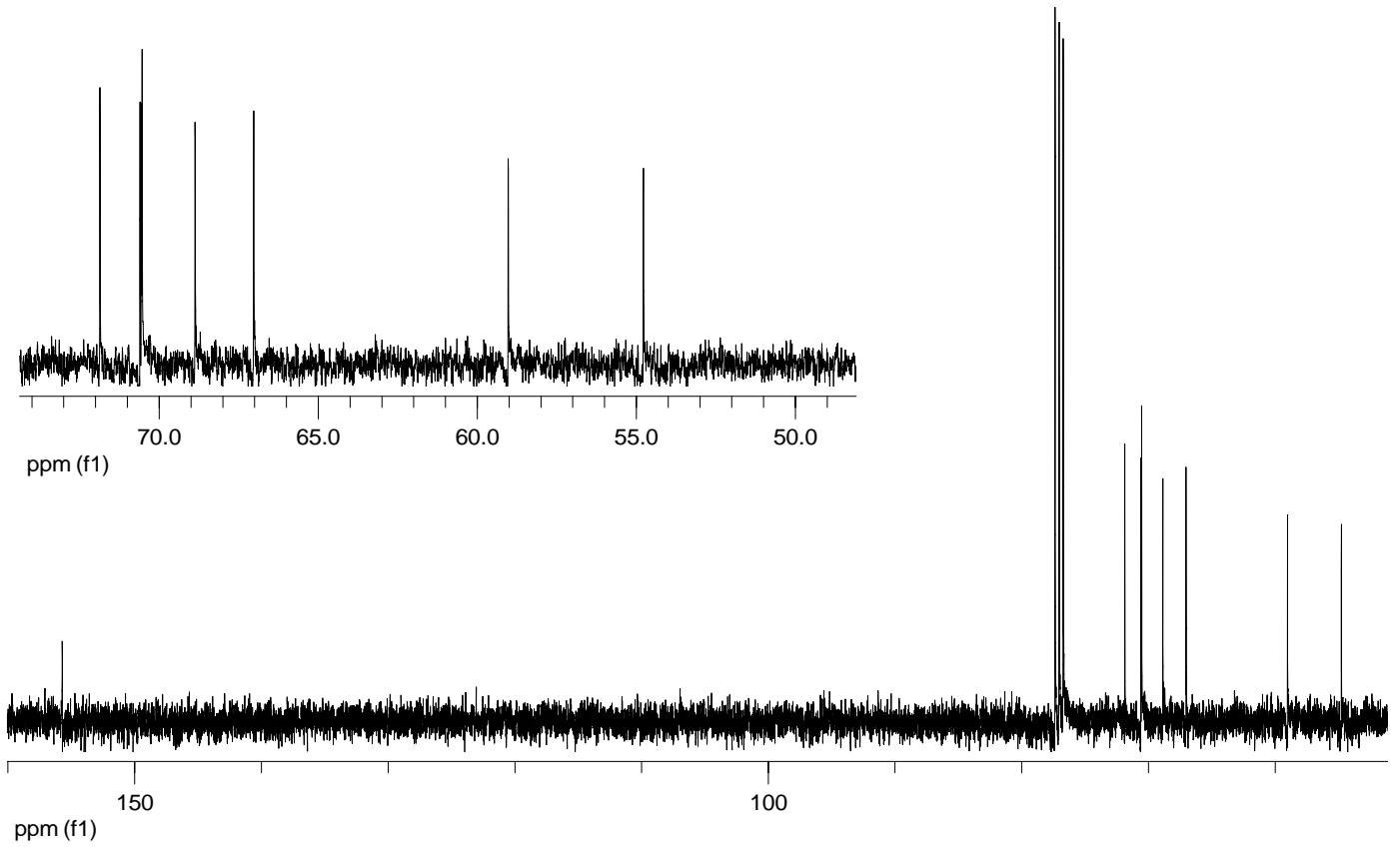

Figure 2. ${ }^{13} \mathrm{C}$ NMR of carbonate 1a

(2-Methoxy)ethyl methyl carbonate, 1c. ${ }^{2}{ }^{1} \mathrm{H}$ NMR (400 MHz, $\left.\mathrm{CDCl}_{3}\right) \delta 3.40(\mathrm{~s}, 3 \mathrm{H}), 3.63(\mathrm{t}$, $2 \mathrm{H}, \mathrm{J}=4.71 \mathrm{~Hz}$ ), 3.80 (s, 3H), $4.31(\mathrm{t}, 2 \mathrm{H}, \mathrm{J}=4.70 \mathrm{~Hz}) .{ }^{13} \mathrm{C} \mathrm{NMR}\left(100 \mathrm{MHz}, \mathrm{CDCl}_{3}\right) \delta$ 54.8, 58.9, 66.8, 70.1, 155. 7. GC-MS, 70 eV, m/z: 134 (M+, <1\%), 103 (11), 77 (18), 59 (85), 58 (100). 

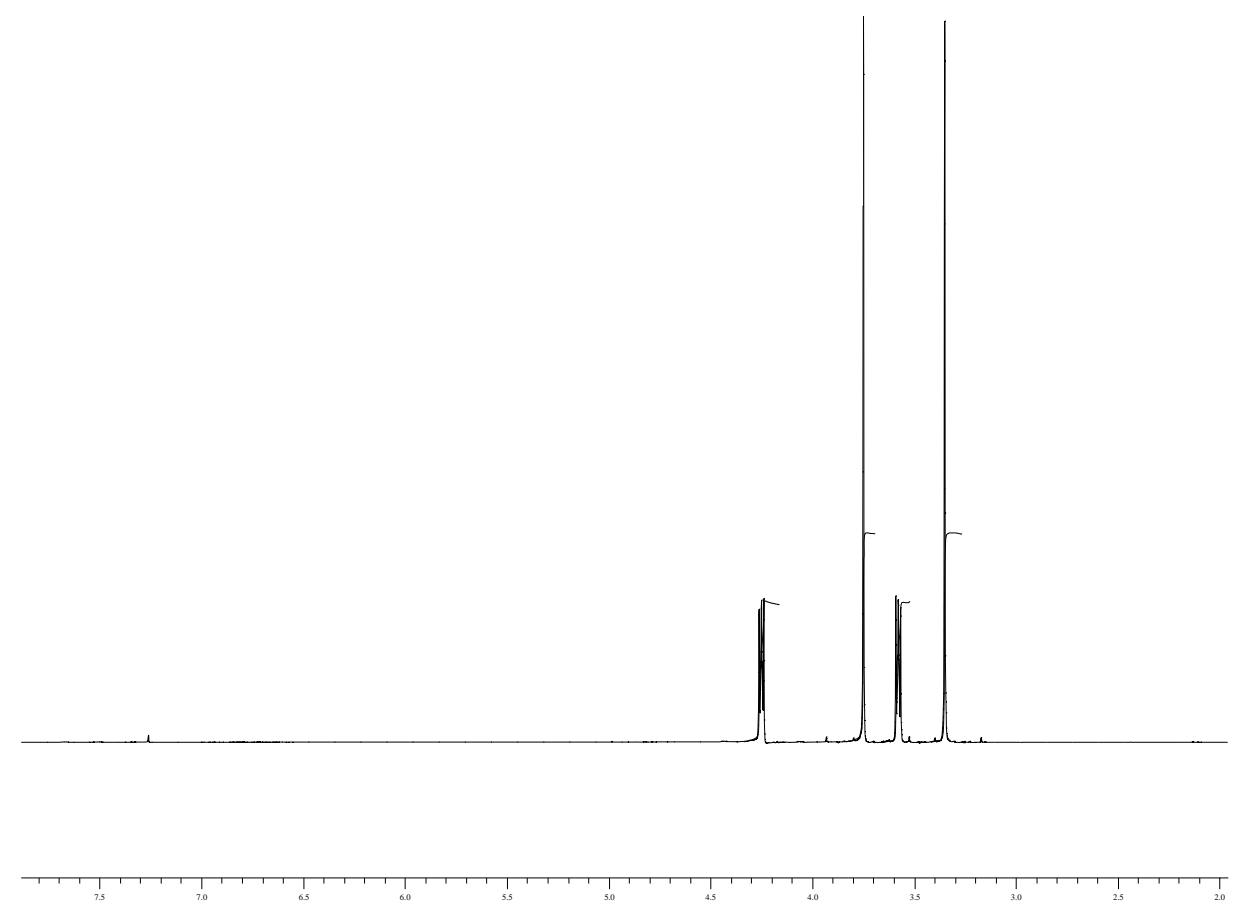

Figure 3. ${ }^{1} \mathrm{H}$ NMR of carbonate 1c

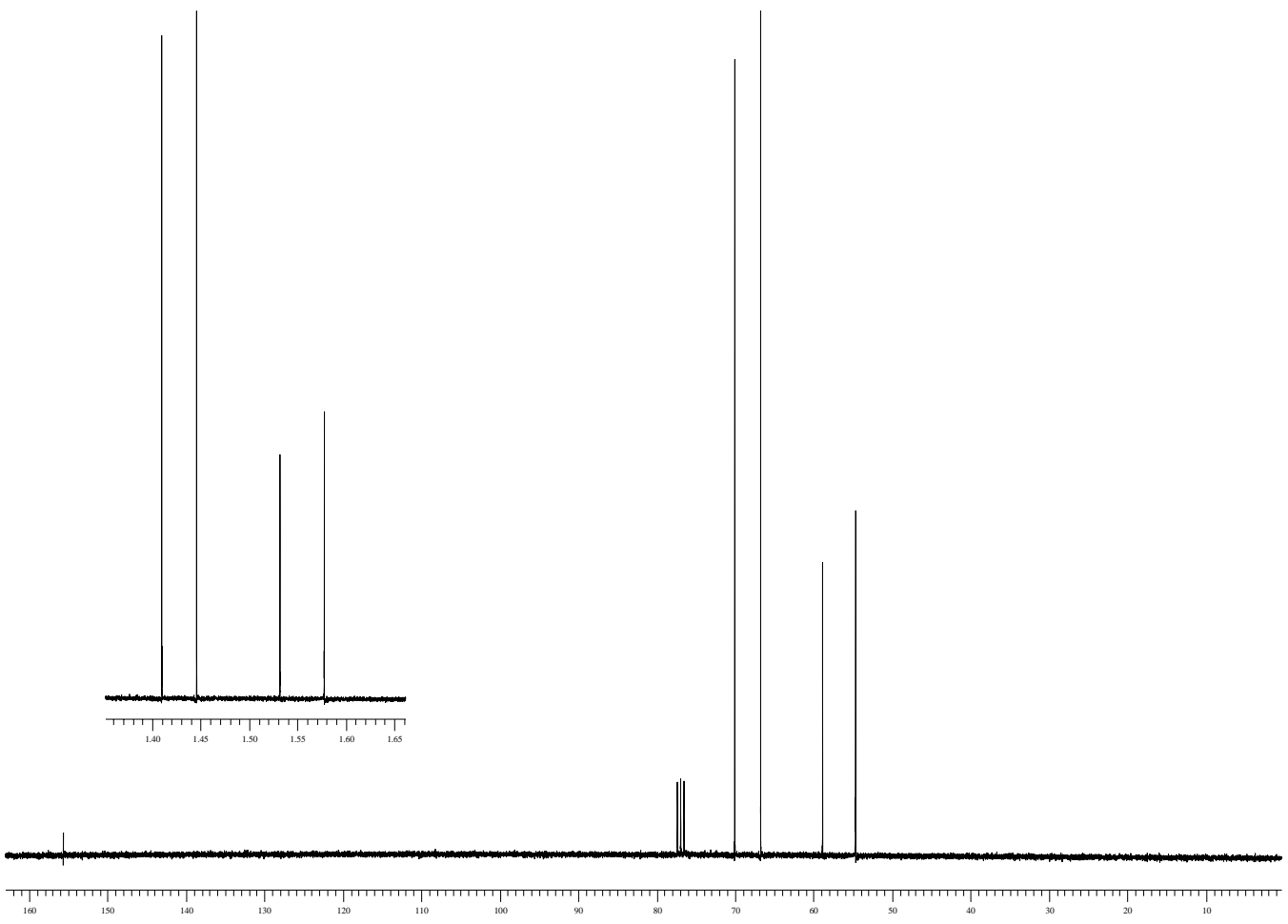

Figure 4. ${ }^{13} \mathrm{C}$ NMR of carbonate 1c 


\section{Isolation and characterisation of $\mathbf{N}, \mathbf{N}$-dimethylanilines $\mathbf{D}_{\mathbf{x}}$.}

N,N-dimethylanilines $\mathbf{D}_{\mathbf{x}}$ were purified by FCC on silica gel F60 (eluant: petroleum ether/diethyl ether in 10:1 v/v), and characterized by ${ }^{1} \mathrm{H}$ NMR (Figures 5-11) and GC/MS. All compounds $\mathbf{D}_{\mathbf{x}}$ were known products whose spectroscopic data were fully reported in the literature. $\mathbf{N , N}$-dimethyl p-anisidine (98\%, by GC): ${ }^{3} \mathrm{mp} 43-45{ }^{\circ} \mathrm{C}$, lit. ${ }^{4} \mathrm{mp} 45-47^{\circ} \mathrm{C} ; \boldsymbol{N}, \boldsymbol{N}$-dimethyl $\boldsymbol{p}$-toluidine $(98 \%$, by GC): ${ }^{3}$ pale yellow liquid, lit. ${ }^{5 a}$ bp $89-89.5^{\circ} \mathrm{C} / 11 \mathrm{~mm}$; $\boldsymbol{N}, \boldsymbol{N}$-dimethyl aniline (99\%, by GC): pale yellow liquid, lit. ${ }^{5 \mathrm{~b}}$ bp $77^{\circ} \mathrm{C} / 13 \mathrm{~mm}$; $\boldsymbol{N}, \boldsymbol{N}$-dimethyl $\boldsymbol{p}$-chloroaniline (97\%, by GC): ${ }^{6} \mathrm{mp} 32-34$ ${ }^{\circ} \mathrm{C}$, lit. ${ }^{5 \mathrm{c}} \mathrm{mp} 35.5^{\circ} \mathrm{C}$; methyl $\boldsymbol{N}, \boldsymbol{N}$-dimethylaminobenzoate $\left(96 \%\right.$, by GC): ${ }^{7} \mathrm{mp} 100-102{ }^{\circ} \mathrm{C}$, lit. ${ }^{5 \mathrm{~d}}$ mp $102{ }^{\circ} \mathrm{C}$; $N, N$-dimethyl o-ethyl aniline (97\%, by GC): ${ }^{8} \mathrm{mp} 132-134{ }^{\circ} \mathrm{C}$, lit. ${ }^{3} 135{ }^{\circ} \mathrm{C}$; $N, N-$ dimethyl 2,3-dimethylaniline (97\%, by GC): ${ }^{9}$ yellow liquid, lit. ${ }^{5 e}$ bp $75{ }^{\circ} \mathrm{C} / 7 \mathrm{~mm}$. The structures of $N, N$-dimethyl $p$-toluidine, $N, N$-dimethyl aniline, $N, N$-dimethyl $p$-chloroaniline, and $N, N$-dimethyl 2,3-dimethylaniline were confirmed also by comparison (GC analyses) to authentic commercial samples.

$\boldsymbol{N}, \boldsymbol{N}$-dimethyl p-anisidine (Figure 5). ${ }^{1} \mathrm{H}$ NMR (300 MHz, $\left.\mathrm{CDCl}_{3}\right) \delta 2.89$ (s, 6H), 3.79 (s, 3H), 3.80 (s, 3H), 6.78 (d, 2H, J = 9.23 Hz), 6.87 (d, 2H, J = 9.04 Hz). 


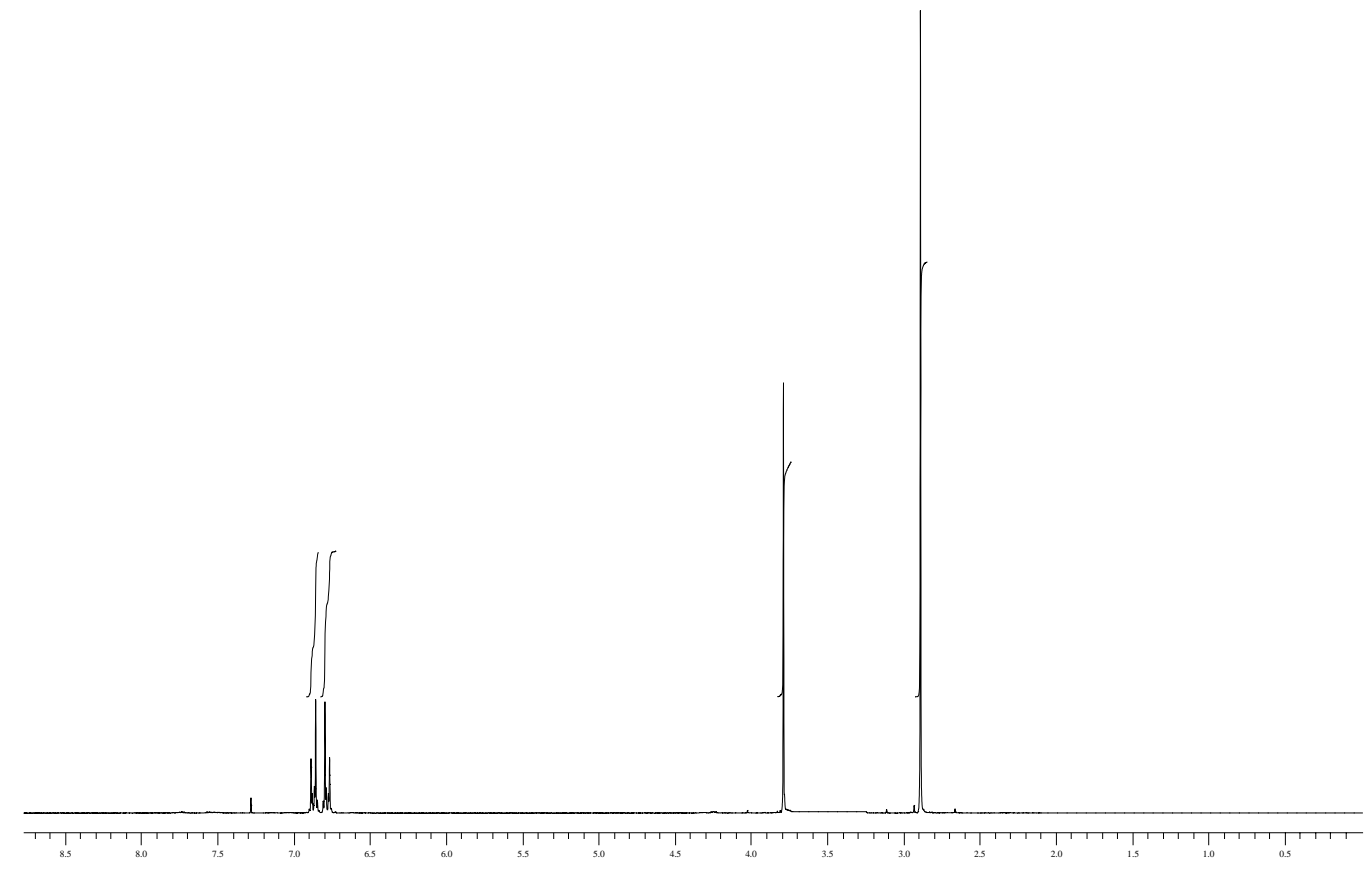

Figure 5. ${ }^{1} \mathrm{H}$ NMR of $N, N$-dimethyl $p$-anisidine

$\boldsymbol{N}, \boldsymbol{N}$-dimethyl p-toluidine (Figure 6). ${ }^{1} \mathrm{H}$ NMR (300 MHz, $\left.\mathrm{CDCl}_{3}\right) \delta 2.25$ (s, 3H), 2.89 (s, 6H), $6.69(\mathrm{~d}, 2 \mathrm{H}, \mathrm{J}=8.85 \mathrm{~Hz}), 7.06(\mathrm{~d}, 2 \mathrm{H}, \mathrm{J}=8.85 \mathrm{~Hz})$.

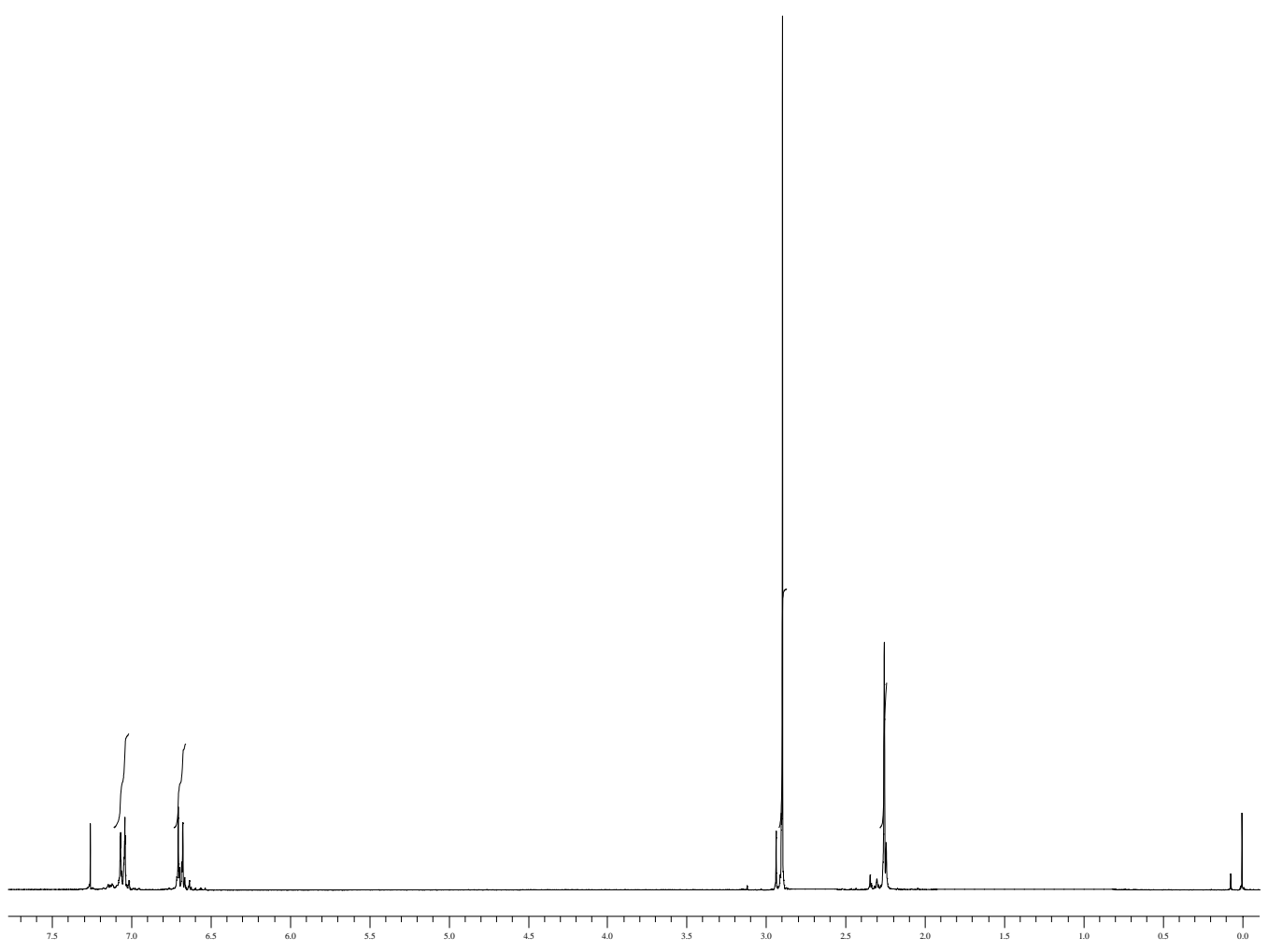

Figure 6. ${ }^{1} \mathrm{H}$ NMR of $N, N$-dimethyl $p$-toluidine 
$\boldsymbol{N}, \boldsymbol{N}$-dimethyl aniline (Figure 7). ${ }^{1} \mathrm{H}$ NMR (300 MHz, $\left.\mathrm{CDCl}_{3}\right) \delta 2.94$ (s, 6H), 6.68-6.77 (m, 3H), 7.20-7.28 (m, 2H).

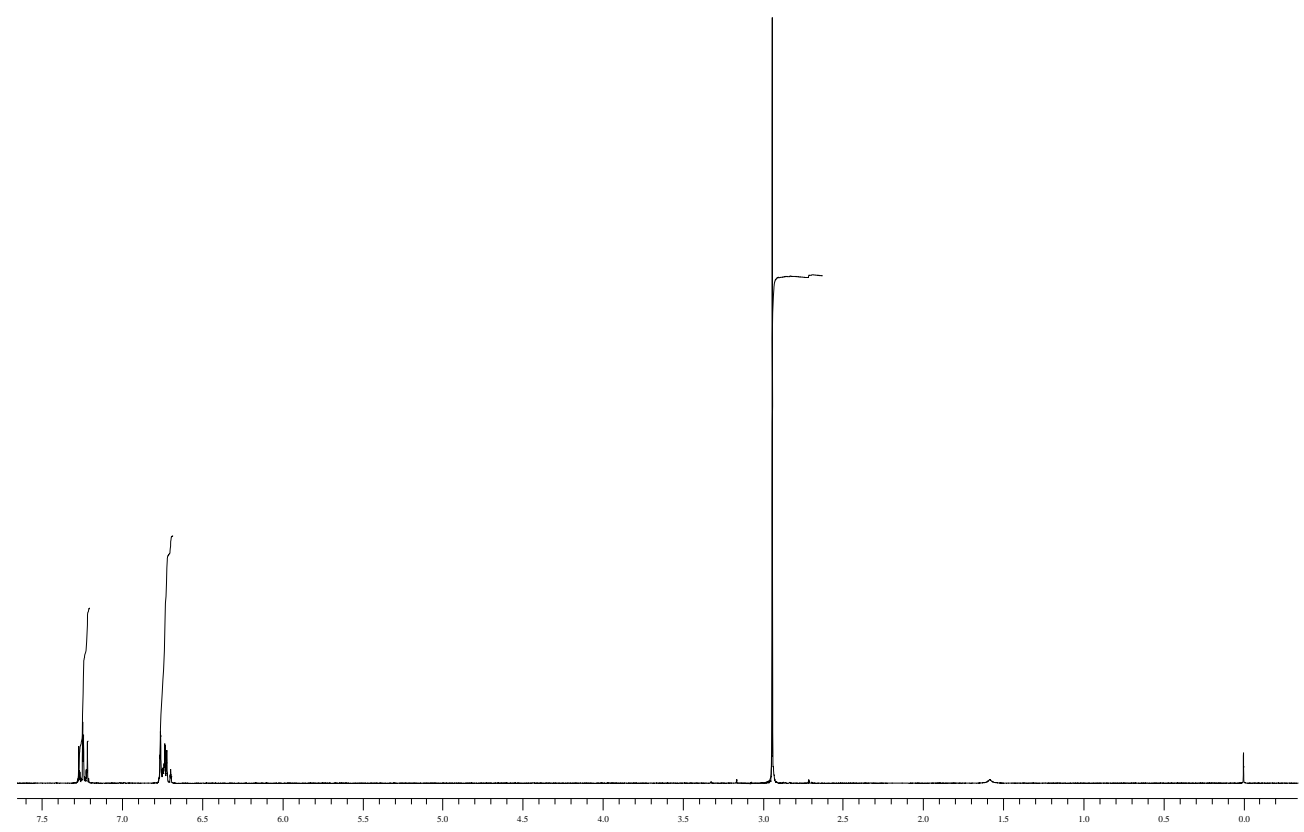

Figure 7. ${ }^{1} \mathrm{H}$ NMR of $N$, $N$-dimethylaniline

$\boldsymbol{N}, \boldsymbol{N}$-dimethyl p-chloroaniline (Figure 8). ${ }^{1} \mathrm{H}$ NMR (300 MHz, $\left.\mathrm{CDCl}_{3}\right) \delta 2.94$ (s, 6H), 6.66 (d, $2 \mathrm{H}, \mathrm{J}=9.23 \mathrm{~Hz}), 7.19(\mathrm{~d}, 2 \mathrm{H}, \mathrm{J}=9.23 \mathrm{~Hz})$.

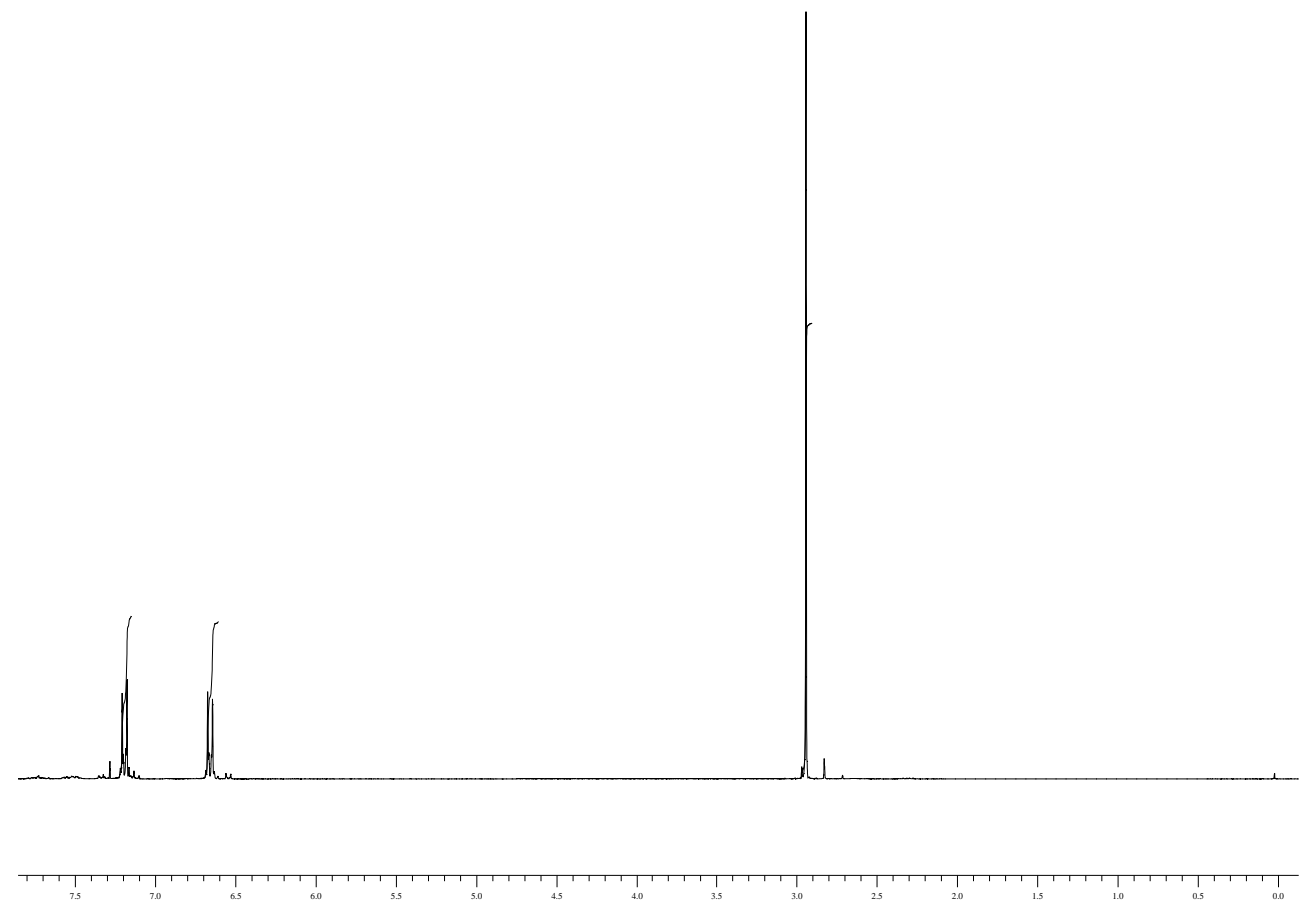

Figure 8. ${ }^{1} \mathrm{H}$ NMR of $N, N$-dimethyl $p$-chloroaniline 
Methyl $\boldsymbol{N}, \boldsymbol{N}$-dimethyl aminobenzoate (Figure 9). ${ }^{1} \mathrm{H}$ NMR (300 MHz, $\left.\mathrm{CDCl}_{3}\right) \delta 3.05$ (s, 6H), 3.87 (s, 3H), 6.66 (d, 2H, J = 9.04 Hz), 7.92 (d, 2H, J = 9.04 Hz).

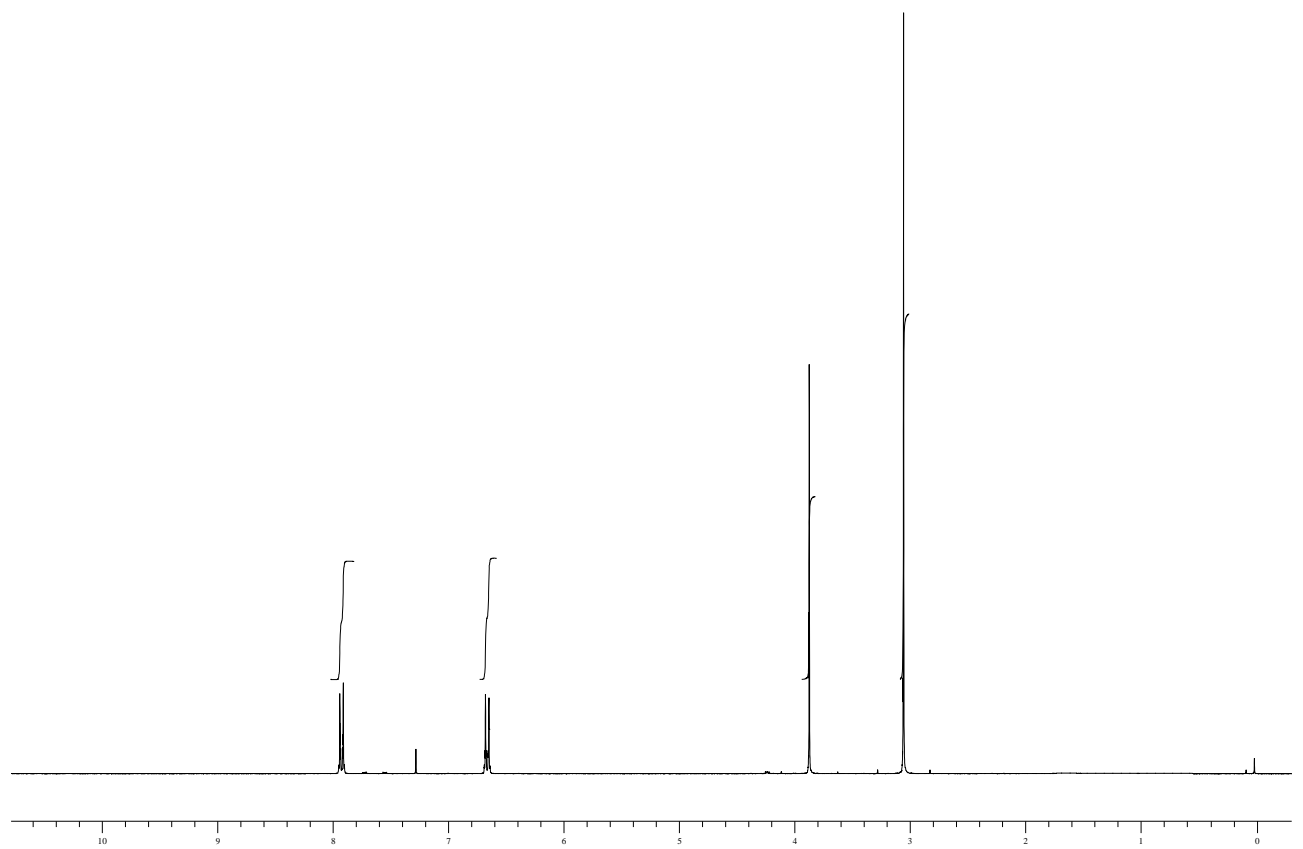

Figure 9. ${ }^{1} \mathrm{H}$ NMR of methyl $N, N$-dimethylaminobenzoate

$\boldsymbol{N}, \boldsymbol{N}$-dimethyl o-ethylaniline (Figure 10). ${ }^{1} \mathrm{H}$ NMR (300 MHz, $\left.\mathrm{CDCl}_{3}\right) \delta 1.25(\mathrm{t}, 3 \mathrm{H}, \mathrm{J}=7.54 \mathrm{~Hz}$ ), 2.68 (s, 6H), 2.73 (q, 2H, J = 7.54 Hz ), 6.99-7.25 (m, 5H).

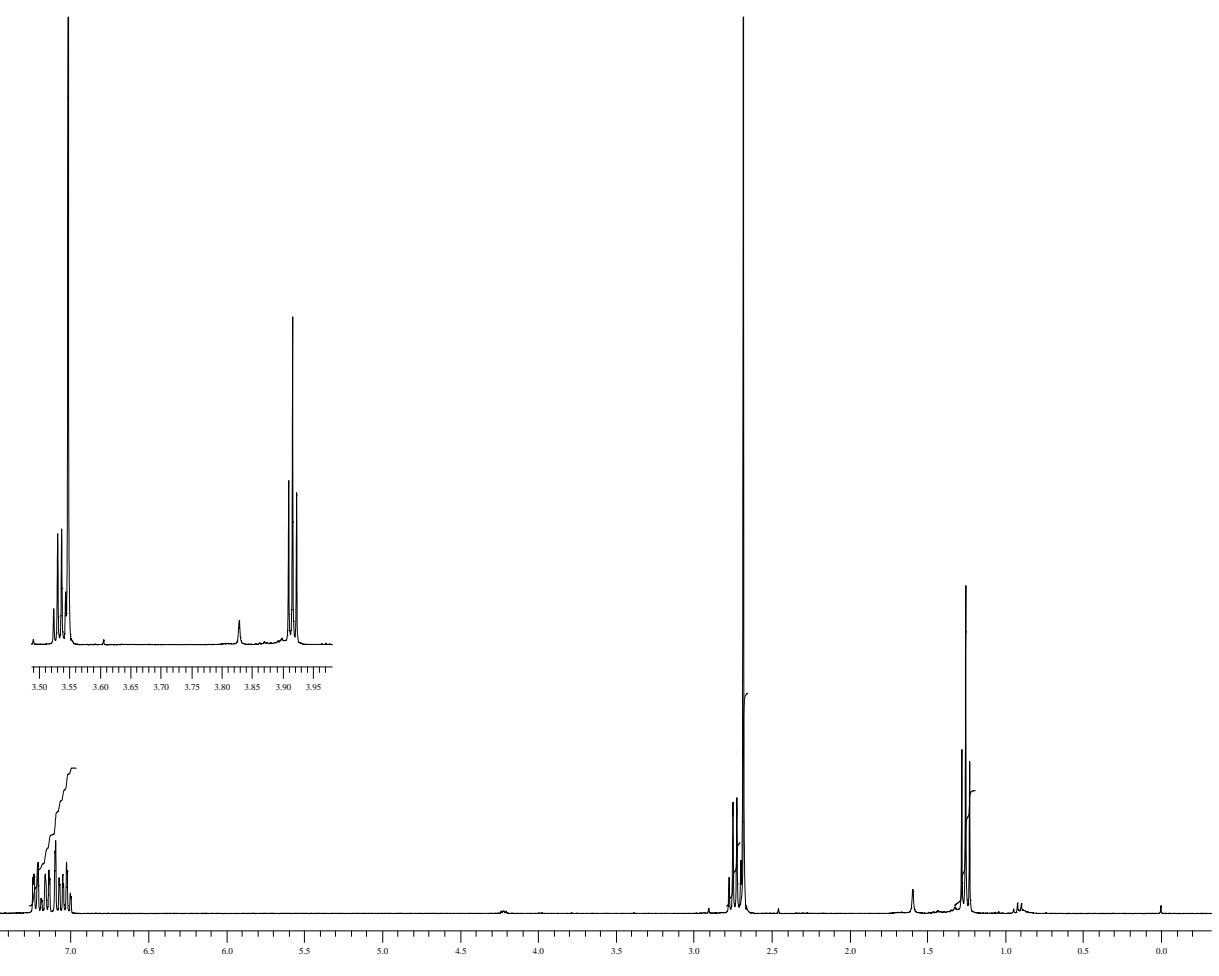


Figure 10. ${ }^{1} \mathrm{H}$ NMR of $N, N$-dimethyl $o$-ethylaniline

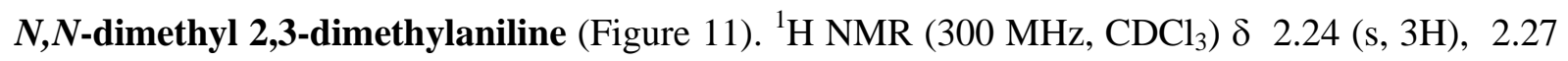
(s, 3H), 2.67 (s, 6H), 6.85-6.95 (m, 2H), 7.03-7.1 (m, 1H).

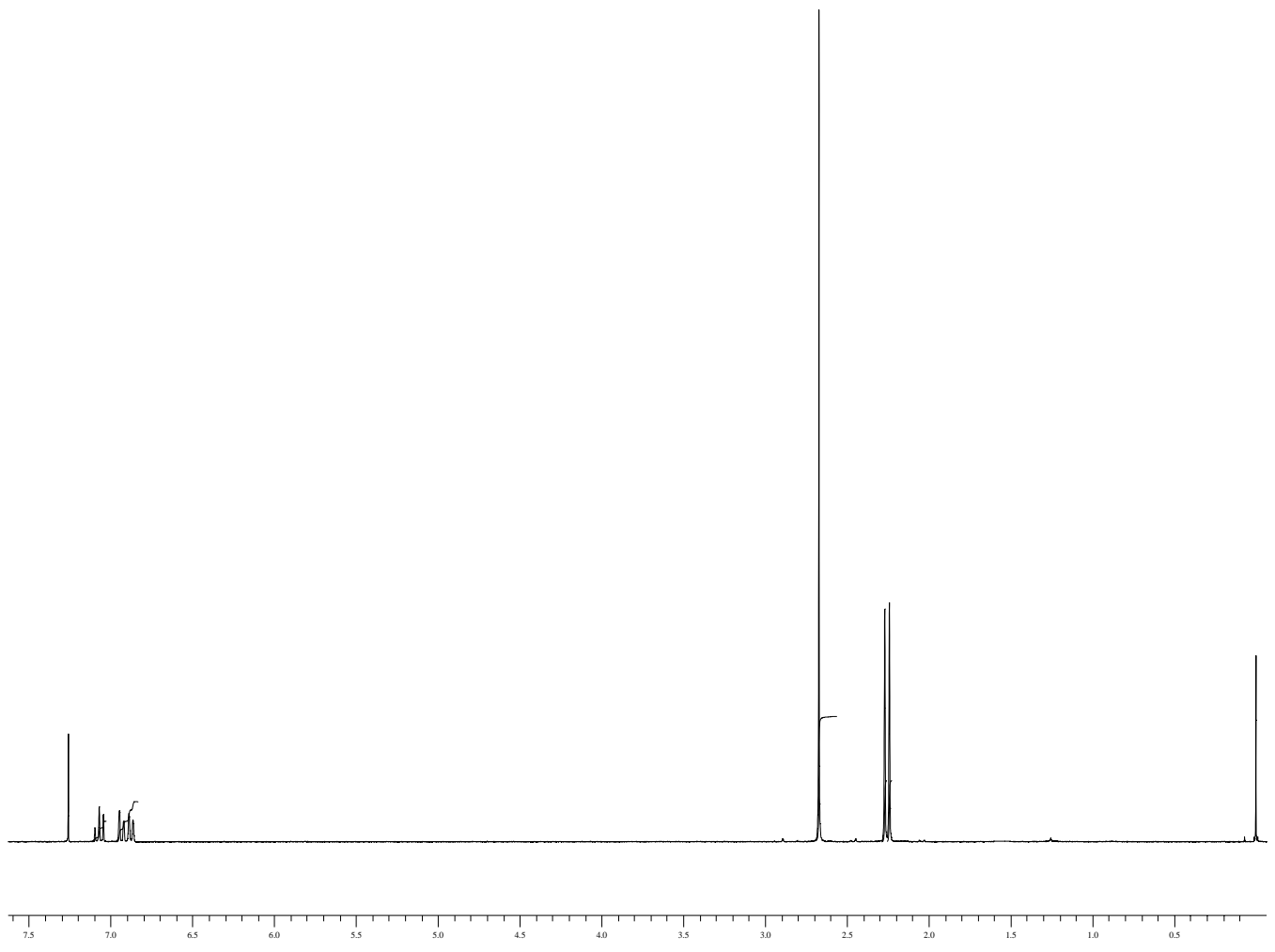

Figure 11. ${ }^{1} \mathrm{H}$ NMR of $N$, $N$-dimethyl 2,3-dimethylaniline

N-Ethyl,N-methyl $\boldsymbol{m}$-toluidine (7b, ${ }^{10}$ Scheme 6) was not isolated from the reaction mixture: its structure was assigned by GC/MS: $149\left(\mathrm{M}^{+}, 38\right), 134\left(\mathrm{M}^{+}-\mathrm{Me}, 100\right), 120$ (M+Et, 4), 119 (15), 118 (14), 91 (24), 65 (12). 


\section{IR Investigations}

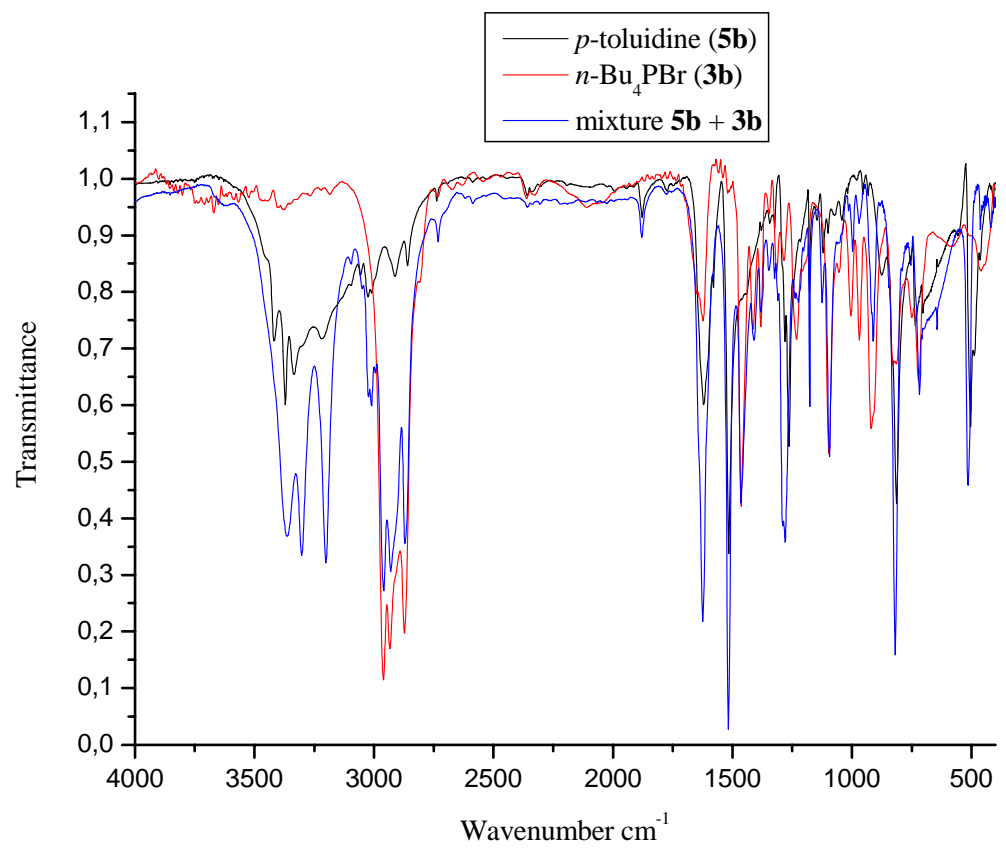

Figure 1. Overlap of IR spectra of pure $p$-toluidine (5b, black), pure $n-\mathrm{Bu}_{4} \mathrm{PBr}$ ( $3 \mathbf{b}$, red), and a mixture of $\mathbf{5 b}$ and $\mathbf{3 b}$ (blue), recorded at room temperature.

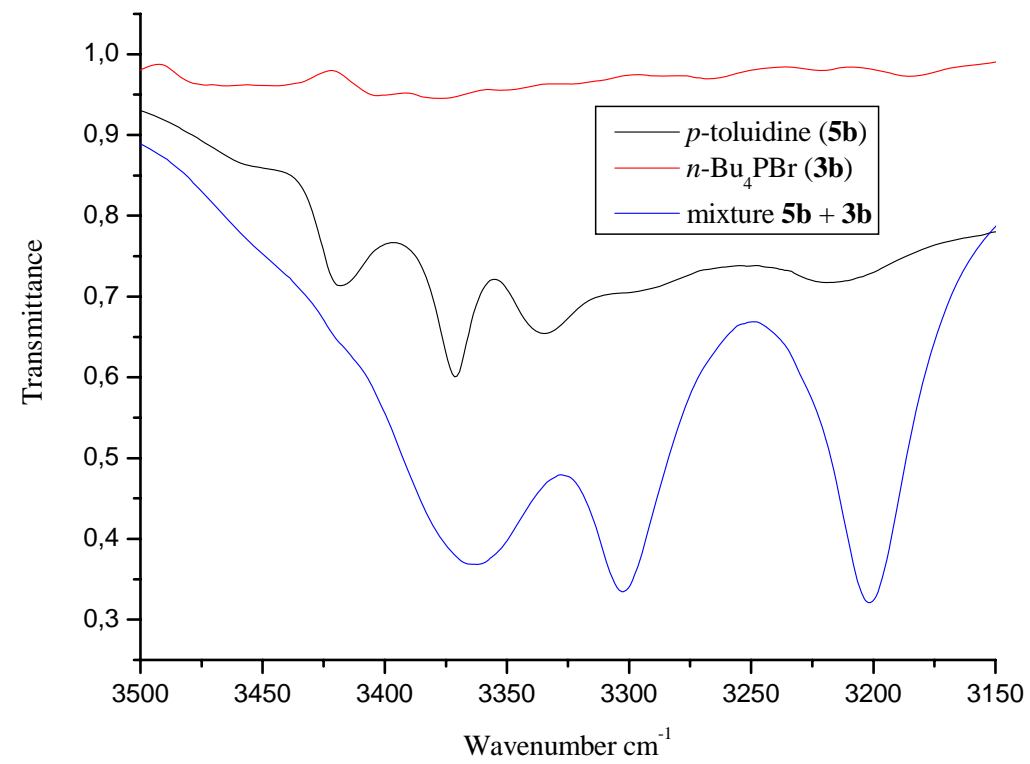

Figure 2. Enlargement of Figure 1 between 3500 and $3150 \mathrm{~cm}^{-1}$. 


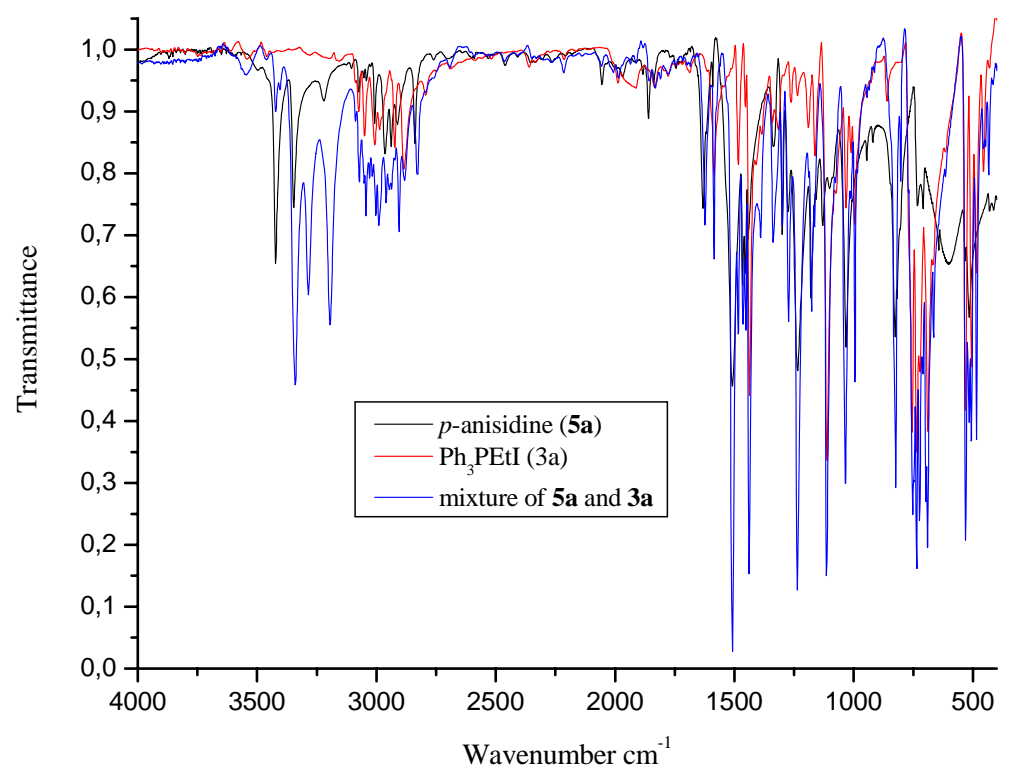

Figure 3. Overlap of IR spectra of pure $p$-anisidine (5a, black), ${ }^{11}$ pure $\mathrm{Ph}_{3} \mathrm{PEtI}$ (3a, red), and a mixture of $5 \mathbf{a}$ and $3 \mathbf{a}$ (blue), recorded at room temperature.

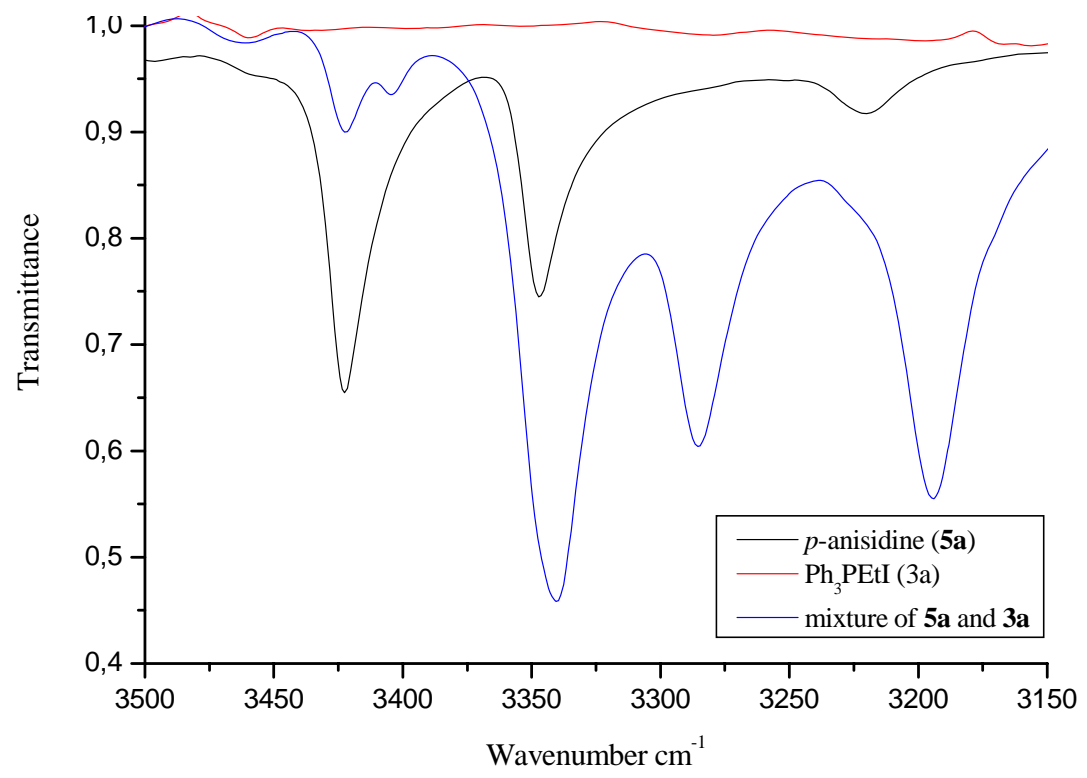

Figure 4. Enlargement of Figure 4 between 3500 and $3150 \mathrm{~cm}^{-1}$.

IR spectra were recorded on commercial p-anisidine (5a), p-toluidine (5b), ethyltriphenyl phosphonium iodide (3a), and tetrabutylphosphonium bromide (3b). Mixtures of $\mathbf{5 a}$ and $\mathbf{3 a}$, of $\mathbf{5 b}$ and $\mathbf{3 a}$, and of $\mathbf{5 b}$ and $\mathbf{3 b}$, were equimolar. 


\section{References}

1 (a) Perosa, A.; Selva, M.; Tundo, P.; Zordan, F. Synlett 2000, 1, 272-274; (b) Selva, M.; Tundo, P.; Perosa; A. J. Org. Chem. 2001, 66, 677-680; (c) Selva, M.; Tundo, P.; Foccardi, T. J. Org. Chem. 2005, 70, 2476-2485.

2 Chuchani, G.; Marquez, E.; Herize, A.; Dominguez, R. M. ; Tosta, M. ; Brusco, D. J. Phys. Org. Chem. 2003, 11, 839-848.

3 Yoo, S.-D.; Tsuno, Y.; Fujo, M.; Sawada, M.; Yukawa, Y. J. Chem. Soc., Perkin Trans. 2, 1989, 7-13.

4 Guarr, T.; McGuire, M. E.; McLendon, G. J. Am. Chem. Soc. 1985, 107, 5104-5111.

5 (a) Dictionary of Organic Compounds, 5th Ed.; Chapman and Hall: New York 1982, Vol. 2, p. 2063; (b) ibid. Vol. 2, p. 2068; (c) ibid. Vol. 1, p. 1055; (d) ibid. Vol. 2, p. 2060; (e) ibid. Vol. 2, p. 2066.

6 (a) Borkowski, W.L.; Wagner, E. C. J. Org. Chem. 1952, 17, 1128-1140; (b) Bhattacharyya, S.; Chatterjee, A.; Duttachowdhury, S.-K. J. Chem. Soc., Perkin Trans. 1, 1994, 1-2.

7 Sim, T. B.; Ahn, J. H.; Yoon, N. M. Synthesis 1996, 324-326

8 Katritzky, A. R.; Rachwal, S., Wu, J. Can. J. Chem. 1989, 68, 456-463.

9 Bertrand, S.; Hofmann, N.; Humbel, S.; Pete, J. P. J. Org. Chem. 2000, 65, 8690-8703.

10 Fahim, H. A.; Galaby, M. J. Chem. Soc. 1950, 3529-3532.

11 Borisenko, E.; Morev, A.V.; Koll, A. J. Mol. Struct. 1998, 444, 183-198. 